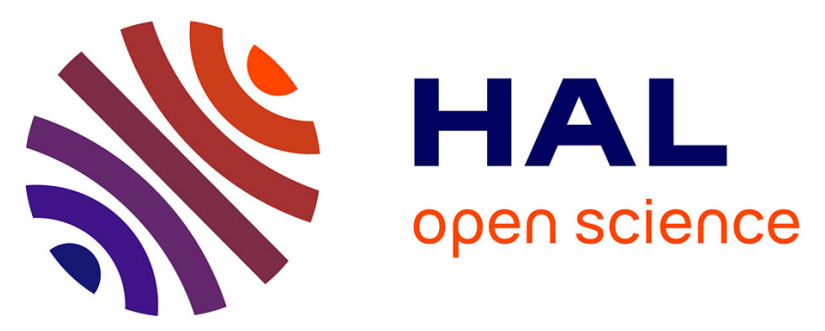

\title{
Atoms, Molecules and Flows: Recent Advances and New Challenges in their Multi-Scale Numerical Modeling at the Beginning of the Third Millenium
}

\author{
Francisco Chinesta, Amine Ammar, Hajer Lamari, Nicolas Ranc
}

\section{- To cite this version:}

Francisco Chinesta, Amine Ammar, Hajer Lamari, Nicolas Ranc. Atoms, Molecules and Flows: Recent Advances and New Challenges in their Multi-Scale Numerical Modeling at the Beginning of the Third Millenium. Innovation in Engineering Computational Technology, Saxe-Coburg Publications, pp.247270, 2008, 10.4203/csets.20.13 . hal-01007478

\section{HAL Id: hal-01007478 https://hal.science/hal-01007478}

Submitted on 8 Sep 2018

HAL is a multi-disciplinary open access archive for the deposit and dissemination of scientific research documents, whether they are published or not. The documents may come from teaching and research institutions in France or abroad, or from public or private research centers.
L'archive ouverte pluridisciplinaire HAL, est destinée au dépôt et à la diffusion de documents scientifiques de niveau recherche, publiés ou non, émanant des établissements d'enseignement et de recherche français ou étrangers, des laboratoires publics ou privés. 


\title{
Atoms, Molecules and Flows: Recent Advances and New Challenges in their Multi-Scale Numerical Modeling at the Beginning of the Third Millenium
}

\author{
F. Chinesta ${ }^{1}$, A. Ammar ${ }^{2}$, H. Lamari ${ }^{2}$ and N. Ranc ${ }^{1}$ \\ ${ }^{1}$ Laboratoire de Mécanique des Systèmes et des Procédés \\ UMR 8106 CNRS-ENSAM, Paris, France \\ ${ }^{2}$ Laboratoire de Rhéologie \\ INPG, UJF, CNRS (UMR 5520), Grenoble, France
}

\begin{abstract}
Nano-science and nano-technology as well as the fine description of the structure and mechanics of materials from the nanometric to the micrometric scales need descriptions ranging from the quantum mechanics to the kinetic theory descriptions characteristic of statistical mechanics. This paper explores the modelling at these scales and points out the main challenges related to the numerical solution of such models that sometimes are discrete but involve an extremely large number of particles (as in the case of molecular dynamics simulations or coarse-grained molecular dynamics) and other times are continuous but they are defined in highly multidimensional spaces leading to the well known curse of dimensionality issues.
\end{abstract}

Keywords: multi-scale modelling, quantum mechanics, molecular dynamics, Brownian dynamics, kinetic theory, statistical mechanics, model reduction, curse of dimensionality, separated representation, finite sums decompositions.

\section{Introduction}

The fine description of the mechanics and structure of materials at the micro, nano and sub-nanometric scales introduces some specific challenges related to the impressive number of degrees of freedom required or the highly dimensional spaces in which those models are defined. Despite the fact that spectacular progress has been accomplished in the context of computational mechanics in the last decade, the treatment of those models, as we describe in the present work, needs further development.

The brute force approach cannot be considered as a possibility for treating this kind of models. Thus, some specialists such as the Nobel Prize R.G. Laughlin, affirmed that no computer existing, or that will ever exist, can break the barriers found in quantum mechanics because it is a catastrophe of dimension [1]. 
We can understand the catastrophe of dimension by assuming a model defined in a hyper-cube in a space of dimension $D, \Omega=]-L, L[D$. Now, if we define a grid to discretize the model, as it is usually performed in the vast majority of numerical methods (finite differences, finite elements, finite volumes, spectral methods etc.), consisting of $N$ nodes on each direction, the total number of nodes will be $N^{D}$. If we assume that for example $N \approx 10$ (an extremely coarse description) and $D \approx 80$ (much lower than the usual dimensions required in quantum or statistical mechanics), the number of nodes involved in the discrete model reaches the astronomical value of $10^{80}$ that represents the presumed number of elementary particles in the universe! We shall come back to the analysis of these systems later.

Thus, progress in this field needs further developments on the physical modelling as well as the introduction of new ideas and methods in the context of computational physics. In this work we are exploring different modelling scales, starting from the finest one, the quantum mechanics, to derive molecular dynamics, Brownian dynamics and finally kinetic theory models. The main particulars of such models, the recent advances and the new challenges will be emphasized.

\section{From quantum mechanics to statistical mechanics: a walk on the frontier of the simulable world}

\subsection{The finest description: the quantum approach}

\subsubsection{The Schrödinger equation}

The quantum state of a given electronic distribution could be determined by solving the Schrödinger equation. This equation has been for a long time considered as one of the finest descriptions of the world. However, before focusing on the challenges of its numerical solution, we would like recall that this equation is not relativistic and that it fails when it is applied to describe heavy atoms. Moreover the Pauli's principle constraint was introduced in the Schrödinger formalism in an ad hoc way, being the reason for the main numerical difficulties.

Some simplified hypotheses are usually introduced, as for example the Born-Oppenheimer that states that the nuclei can be in a first approximation assumed as classical point-like particles, that the state of electrons only depends on the nuclei positions and that the electronic ground state corresponds to the one that minimizes the electronic energy for a nuclear configuration. This equation defines a multidimensional problem whose dimension increases linearly with the number of the electrons in the system.

Thus, the knowledge of a quantum system reduces to the determination of the wavefunction $\Psi\left(\mathbf{x}_{1}, \mathbf{x}_{2}, \cdots, \mathbf{x}_{N}, t ; \mathbf{X}_{1}, \cdots, \mathbf{X}_{M}\right)$ (that establishes that the electronic wavefunction depends parametrically on the nuclei positions) whose evolution is governed 
by the Schrödinger equation:

$$
i \hbar \frac{\partial \Psi}{\partial t}=-\frac{\hbar^{2}}{2 m_{e}} \sum_{e=1}^{e=N} \nabla_{e}^{2} \Psi+\sum_{e=1}^{e=N-1} \sum_{e^{\prime}=e+1}^{e^{\prime}=N} V_{e e^{\prime}} \Psi+\sum_{e=1}^{e=N} \sum_{n=1}^{n=M} V_{e n} \Psi
$$

where $N$ is the number of electrons and $M$ the number of nuclei, the last ones assumed located and fixed at positions $\mathbf{X}_{j}$. Each electron is defined in the whole physical space $\mathbf{x}_{j} \in \mathbf{R}^{3}, i=\sqrt{-1}, \hbar$ represents the Planck's constant divided by $2 \pi$ and $m_{e}$ is the electron mass.

The differential operator $\nabla_{e}^{2}$ is defined in the conformation space of each particle, i.e.: $\nabla_{e}^{2} \equiv \partial^{2} / \partial x_{e}^{2}+\partial^{2} / \partial y_{e}^{2}+\partial^{2} / \partial z_{e}^{2}$. The Coulomb's potentials accounting for the electron-electron and electron-nuclei interactions are written as:

$$
\begin{gathered}
V_{e e^{\prime}}=\frac{\left(q_{e}\right)^{2}}{\left\|\mathbf{x}_{e}-\mathbf{x}_{e^{\prime}}\right\|} \\
V_{e n}=-\frac{q_{n} q_{e}}{\left\|\mathbf{x}_{e}-\mathbf{X}_{n}\right\|}
\end{gathered}
$$

The electron charge is represented by $q_{e}$ and the nuclei charge by $q_{n}=\left|q_{e}\right| \times Z$ (where $Z$ is the atomic number).

The time independent Schrödinger equation (from which one could determine the ground state, perform quantum static computations or accomplish separated representations of the time-dependent solution) is written:

$$
-\frac{\hbar^{2}}{2 m_{e}} \sum_{e=1}^{e=N} \nabla_{e}^{2} \Psi+\sum_{e=1}^{e=N-1} \sum_{e^{\prime}=e+1}^{e^{\prime}=N} V_{e e^{\prime}} \Psi+\sum_{e=1}^{e=N} \sum_{n=1}^{n=M} V_{e n} \Psi=E \Psi
$$

where the ground state corresponds to the eigenfunction $\Psi_{0}$ associated with the most negative eigenvalue $E_{0}$.

\subsubsection{Advanced models: The Dirac equation}

The Schrödinger equation does not agree with the relativity principle (it does not verify the Lorentz transformation). Considering the relativistic expression of the Hamiltonian

$$
H^{2}=p^{2} c^{2}+m^{2} c^{4}
$$

one could derive the so-called Klein-Gordon equation:

$$
-\hbar^{2} \frac{\partial^{2}}{\partial t^{2}} \Psi=\left(-\hbar^{2} c^{2} \nabla^{2}+m^{2} c^{4}\right) \Psi
$$

that has been widely used to model bosonic systems. However, even if it agrees with the relativity constraints, it cannot ensure a positive definite electronic distribution. 
To circumvent these difficulties, in 1928 Dirac proposed an alternative approach that agrees with the relativity constraints and leads to a positive definite electronic distribution. The Dirac equation for a system composed of a single particle states:

$$
i \hbar \frac{\partial}{\partial t} \Psi=-i \hbar c\left(\hat{\alpha}_{x} \frac{\partial \Psi}{\partial x}+\hat{\alpha}_{y} \frac{\partial \Psi}{\partial y}+\hat{\alpha}_{z} \frac{\partial \Psi}{\partial z}\right)+\beta m c^{2} \Psi=H_{e} \Psi
$$

where the quadrivector $\Psi$ represents the so-called spinnor, that contains for the particle and the associated anti-particle the distribution for both spin values, and $H_{e}$ denotes the one particle Hamiltonian.

When this equation is written in the Hartree atomic unit system, in which $\hbar=1$, $m=1, c=137$, and applied for a one particle system within a potential $V$, the ground-state steady solution results from the solution of the eigenproblem:

$$
-i c\left(\hat{\alpha}_{x} \frac{\partial \Psi}{\partial x}+\hat{\alpha}_{y} \frac{\partial \Psi}{\partial y}+\hat{\alpha}_{z} \frac{\partial \Psi}{\partial z}\right)+\beta c^{2} \Psi+V(x, y, z) \Psi=E \Psi
$$

where the different matrices are defined by:

$$
\begin{aligned}
\beta & =\left(\begin{array}{cccc}
1 & 0 & 0 & 0 \\
0 & 1 & 0 & 0 \\
0 & 0 & -1 & 0 \\
0 & 0 & 0 & -1
\end{array}\right) \\
\hat{\alpha}_{x} & =\left(\begin{array}{cccc}
0 & 0 & 0 & 1 \\
0 & 0 & 1 & 0 \\
0 & 1 & 0 & 0 \\
1 & 0 & 0 & 0
\end{array}\right) \\
\hat{\alpha}_{y} & =\left(\begin{array}{cccc}
0 & 0 & 0 & -i \\
0 & 0 & -i & 0 \\
0 & i & 0 & 0 \\
i & 0 & 0 & 0
\end{array}\right) \\
\hat{\alpha}_{z} & =\left(\begin{array}{cccc}
0 & 0 & 1 & 0 \\
0 & 0 & 0 & -1 \\
1 & 0 & 0 & 0 \\
0 & -1 & 0 & 0
\end{array}\right)
\end{aligned}
$$

For a system composed of $N_{e}$ electrons and $N_{n}$ nuclei the atomic potential states:

$$
V=\sum_{e=1}^{e=N_{e}} \sum_{n=1}^{n=N_{n}} V_{e n}+\sum_{e=1}^{e=N_{e}-1} \sum_{e^{\prime}=e+1}^{e^{\prime}=N_{e}} V_{e e^{\prime}}
$$

where 


$$
V_{e e^{\prime}}=\frac{1}{\left\|\mathbf{x}_{e}-\mathbf{x}_{e^{\prime}}\right\|}-\frac{\hat{\alpha}_{x e} \hat{\alpha}_{x e^{\prime}}}{\left\|\mathbf{x}_{e}-\mathbf{x}_{e^{\prime}}\right\|}-\frac{\hat{\alpha}_{y e} \hat{\alpha}_{y e^{\prime}}}{\left\|\mathbf{x}_{e}-\mathbf{x}_{e^{\prime}}\right\|}-\frac{\hat{\alpha}_{z e} \hat{\alpha}_{z e^{\prime}}}{\left\|\mathbf{x}_{e}-\mathbf{x}_{e^{\prime}}\right\|}
$$

and

$$
V_{e n}=-\frac{Z}{\left\|\mathbf{x}_{e}-\mathbf{X}_{n}\right\|}
$$

where $Z$ is the atomic number.

Thus, the eigenproblem to be solved reads:

$$
\underbrace{\left(\sum_{e=1}^{e=N_{e}} H_{e}+\sum_{e=1}^{e=N_{e}} \sum_{n=1}^{n=N_{n}} V_{e n}+\sum_{e=1}^{e=N_{e}-1} \sum_{e^{\prime}=e+1}^{e^{\prime}=N_{e}} V_{e e^{\prime}}\right)}_{\mathcal{H}} \Psi=E \Psi
$$

where $H_{e}$ and $\mathcal{H}$ denote the single particle and the whole system Hamiltonians respectively. The ground-state is given by the spinnor $\Psi$ related to the lowest positive eigenvalue $E_{g s}$

Despite the fact that the Dirac formulation can address systems composed of heavy nuclei, whose treatment needs the consideration of relativistic effects, the Pauli's exclusion principle must be enforced explicitly as was the case within the Schrödinger framework. Thus, the antisymmetry of $\Psi$ must be enforced by using, for example, the Slater determinant.

Thus, in what follows, we are focussing on the numerical solution of the Schrödinger equation, because the vast majority of techniques that were applied for solving the Schrödinger equation were then extended for solving the Dirac one.

\subsubsection{On the numerical solution of the Schrödinger equation}

Several techniques have been proposed for solving the Schrödinger equation. Some of them lie in the direct solution of the (time-independent or time-dependent) Schrödinger equation. Due to the curse of dimensionality its solution was only possible for very reduced populations of electrons.

Another solution strategy is based on the Hartree-Fock (HF) approach and its derived approaches (post-Hartree-Fock methods). The main assumption of this approach lies in the approximation of the joint electronic wavefunction (related to the $N$ electrons) as a product of $N 3 D$-functions (the molecular orbitals) verifying the antisymmetry restriction derived from the Pauli's principle. Thus, the original HF approach consists of writing the joint wavefunction from a single Slater's determinant. The Schödinger equation allows computing the $N$ molecular orbitals after solving the resulting strongly non-linear problem. This technique has been extensively used in quantum chemistry to analyze the structure and behavior of molecules involving a moderate number of electrons. Of course, the HF assumption sometimes represents too crude an approximation which invalidates the derived results. 
To circumvent this crude approximation different multi-determinant approaches have been proposed. Interested readers can refer to the excellent overview of Cancès et al. [2] as well as the different chapters of the handbook on computational chemistry [3]. The simplest possibility consists in writing the solution as a linear combination of some Slater determinants built by combining $n$ molecular orbitals, with $n>N$. These molecular orbitals are assumed to be known (e.g. the orbitals related to the hydrogen atom) and the weights are searched to minimize the electronic energy. When the molecular orbitals are built from the Hartree-Fock solution (by employing the ground state and some excited eigenfunctions) the technique is known as Configuration Interaction method (CI). A more sophisticated technique consists in writing this many-determinants approximation of the solution by using a number of molecular orbitals $n$ (with $n>N$ ) assumed unknown. Thus, the minimization of the electronic energy leads the molecular orbitals as well as the associated coefficients of this many-determinants expansion to compute simultaneously. Obviously, each one of these unknown molecular orbitals are expressed in an appropriate functional basis (e.g. gaussian functions). This strategy is known as Multi-Configuration Self-Consistent Field (MCSCF).

All the strategies just mentioned (and others like the coupled cluster or the MollerPlesset perturbation methods) belong to the family of the wavefunction based methods. In any case all these methods can be used only to solve quantum systems composed of a moderate number of electrons. As we confirm later the main difficulty is not in the dimensionality of the space, but in the use of the Slater determinants (needed to account for the Pauli's principle) whose complexity scales on the factorial of the number of electrons, i.e. in $N$ !.

The second family of approximation methods, widely used in quantum systems composed of hundreds, thousands and even millions of electrons, are based on the density functional theory (DFT). These models, more than looking for the expression of the wavefunction (with the associated multi-dimensional issue) look for the electronic distribution $\rho(\mathbf{x})$ itself. The main difficulties of this approach are related to the expressions of both the kinetic energy of electrons and the inter-electronic repulsion energy. The second term is usually modelled from the electrostatic self-interaction energy of a charge distribution $\rho(\mathbf{x})$. On the other hand the kinetic energy term is also evaluated in an approximate manner (from the electronic distribution itself in the Thomas-Fermi and related orbital-free DFT models or from a system of $N$ noninteracting electrons - Kohn-Sham models). Obviously, due to the just mentioned approximations introduced in the kinetic and inter-electronic interaction energies, a correction term is needed, the so-called exchange-correlation-residual-kinetic energy. However, no exact expression of this correction term exists and then different approximate expressions have been proposed and used. Thus, the validity and accuracy of the computed results will depend on the accuracy of the the exchange-correlation term that must be fitted for each system.

The models related to the Thomas-Fermi, less accurate in practice because of the too phenomenological expression of the kinetic energy coming from the reference 
system of a uniform non-interacting electron gas, allows one to consider large multielectronic systems. In a recent work, Gavini et al. [4] performed multi-million atom simulations by employing the Thomas-Fermi-Weizsacker family of orbital-free kinetic energy functionals. On the other hand, the Kohn-Sham based models are a priori more accurate, but they need the computation of the $N$ eigenfunctions related to the $N$ lowest eigenvalues of a non-physical atom composed of $N$ non-interacting electrons.

Transient solutions are very common in the context of quantum gas dynamics (physics of plasma) but are more infrequent in material science when the structure and properties of molecules or crystals are concerned. For this reason, in what follows, we are focusing on the solution of the time-independent Schrödinger equation which leads to the solution of the associated multidimensional eigenproblem, whose eigenfunction related to the most negative eigenvalue constitutes the ground state of the system.

Quantum chemistry calculations performed in the Born-Oppenheimer setting consist either (i) in solving the geometry optimization problem, that is, to compute the equilibrium molecular configuration (nuclei distribution) that minimizes the energy of the system, finding the most stable molecular configuration that determines numerous properties like for instance infrared spectrum or elastic constants; or (ii) in performing an ab initio molecular dynamics simulation, that is, to simulate the time evolution of the molecular structure according to the Newton law of classical mechanics. Molecular dynamics simulations allow computation of various transport properties (thermal conductivity, viscosity, etc.) as well as some other non-equilibrium properties.

\subsection{From "ab initio" to molecular dynamics}

Depending on the choice of the method, on the accuracy required, and on the computer facility available, the $a b$ initio methods allow today for the simulation of systems up to ten, one hundred or some million atoms. In time dependent simulations, they are only convenient for small-time simulations, say not more than a picosecond. However, sometimes larger systems are concerned, and for this purpose one must focus on faster approaches, obviously less accurate. Two possibilities exist: the semi-empirical and the empirical approaches. The semi-empirical approaches speed up the $a b i n i$ tio methods by profiting from the information coming from experiments or previous simulations. Empirical methods go on by considering explicitly only the nuclei, by introducing "empirical" potentials leading to the forces acting on the nuclei. Thus, in the stationary setting only the stable configuration is searched, and for this a geometrical optimization (to computed the nuclei equilibrium distribution) is addressed leading to the so-called molecular mechanics. The transient setting results in the classical molecular dynamics but now the computation is speeded up by many orders of magnitude with respect to the molecular dynamics where the potentials are computed at the ab initio level.

Thus, if we assume a population of $M$ nuclei (of mass $m_{n}$ ) and a two-body potential (many-body potentials are also available), now the Newton's law writes for a generic 
nuclei $n$ :

$$
m_{n} \frac{d^{2} \mathbf{X}_{n}}{d t^{2}}=\sum_{k=1, k \neq n} \mathbf{F}_{k}^{n}, \quad \forall n \in[1, \cdots, M]
$$

where $F_{k}^{n}$ denotes the force acting on nucleus $n$ originated by the presence of nucleus $k$. Obviously these forces can be computed from the gradient of the assumed interparticle potentials.

Accurate algorithms for integrating these equations exist. The simplectic Verlet's scheme is one of the most used. Molecular dynamics simulations are confronted, despite their conceptual simplicity, with diverse difficulties of different nature:

- The first and most important comes, as previously indicated, from the impossibility of using an "exact" interaction potential derived from quantum mechanics. This situation is particularly delicate when we are dealing with some irregular nuclei distributions such as the ones encountered in the neighborhood of defaults in crystals (dislocations, crack tips, etc.), interfaces between different materials or in zones where different kinds of nuclei coexist.

- The second one comes from the units involved in this kind of simulations: the nuclei displacements are in the nanometric scale, the energies are of the order of the electron-volts, the time steps are of the order of $10^{-15} \mathrm{~s}$. Thus, because of the limits in the computer's precision, a change of units is required, which can be easily performed.

- In molecular dynamics the behavior of atoms and molecules is described in the framework of classical mechanics. Thus, the particles energy variations are continuous. The applicability of MD depends on the validity of this fundamental hypothesis. When we consider crystals at low temperature the quantum effects (implying discontinuous energy variations) are preponderant, and in consequence the matter properties at these temperatures cannot be determined by MD simulations. The use of MD is restricted to temperatures higher than the Debye's temperature (for example the Debye's temperature for the $\mathrm{Fe} \alpha \approx 460 K$ ). This analysis is in contrast to the vast majority of MD simulations carried out nowadays. In fact, higher is the temperature (kinetic energy) and higher results the velocity of particles, requiring shorter time steps in order to ensure the stability of the integration scheme. For this reason, nowadays most of the MD simulations in solid mechanics are carried out a zero degrees Kelvin or at very low temperatures but, as just pointed out, at these temperatures the validity of the computed MD solutions are polluted by the non negligible quantum effects.

- The prescription of boundary conditions is another delicate task. If the analysis is restricted to systems with free boundary conditions, then the MD simulation can be carried out without any particular treatment. On the other hand we must consider a system large enough to ensure that in the analyzed region the impact 
of the free surfaces can be neglected. Another possibility lies in the prescription of periodic boundary conditions, where an atom leaving the system for example through the right boundary is re-injected in the domain through the left boundary. Moreover, the particles located in the neighborhood of a boundary are influenced by the ones located in the neighborhood of the opposite boundary. The imposition of other boundary conditions is more delicate from both the numerical and the conceptual points of view. For example, what is the meaning of prescribing a displacement on a boundary? Each situation requires a deep analysis in order to define the best (the most physical) way to prescribe the boundary conditions.

- There are other difficulties related to the transient analysis, including the prescription of the initial conditions. Let a thermal system be in equilibrium (where the velocities distribution is in agreement with the Maxwell-Boltzmann distribution). Now, we proceed to heat the system. One possibility lies in increasing suddenly the kinetic energy of each particle. Obviously, even if the resulting velocities define a Maxwell-Boltzmann's distribution, the system remains off equilibrium because the partition between kinetic and potential energies is not the appropriate one. For this reason we must proceed to relax the system that evolves from this initial (non-physical) state to the equilibrium one. Other (more physical) possibility lies in the incorporation of a large enough ambient region around the analyzed system, whose particles are initially in equilibrium at the highest temperature. Now, both regions (the system and the ambient) interact, and the system initiates its heating process that reaches its equilibrium some time later. The final state of both evolutions is the same, but the time required to reach it depends on the technique used to induce the heating. The first transient is purely artificial whereas the second one is more physical allowing the identification of some transport coefficients (for example the thermal conductivity).

- Finally the CPU time continues to be the main limitation of MD simulations. The strongest handicap is related to the necessity of considering at each time step and for each particle the influence of all the others particles. Thus, the integration method seems to scale with the square of the number of particles. Even if some computing time savings can be introduced in the neighbors search, the extremely small time steps and the extremely large number of particles required to describe real scenarios, limit considerably the range of applicability of this kind of simulation, that has been accepted to be nowadays, in 2007, of the order of a cubic micrometer, even when the systems are considered at very low, and then non-physical, temperatures (close to zero degrees Kelvin). We can notice that, despite the impressive advances in the computational availabilities, the high performance computing and the use of massive parallel computing platforms, the state of the art does not allow the treatment of macroscopic systems encountered in practical applications of physics, chemistry and engineering.

The above mentioned difficulties in performing fully molecular dynamics simula- 
tions led to hybrid techniques that apply MD in the regions where the unknown field varies in a non-uniform way (molecular dynamics model) and a standard finite element approximation in those regions where the unknown field variation can be considered as uniform (continuous model). The main questions raised by these bridging strategies concern: (i) the kinematics representations in both models; (ii) the transfer conditions on the MD and continuous models interface and (iii) the macroscopic constitutive equation employed in the continuous model.

Different alternatives exist, and the construction of such bridges is nowadays one of the most active topics in computational mechanics. The spurious reflection of the high frequency parts of the waves is one of the main issues. We would like only to mention three "families" of bridging techniques, giving some key references in which the interested reader could find other extremely valuable references: (i) the quasi-continuum method proposed by Tadmor and Ortiz [5]; (ii) the multi-scale method proposed by Wagner and Liu [6] and (iii) the methods based on the "Arlequin" approach [7] like the one proposed by Belytschko in [8].

\subsection{Coarse grained modelling: Brownian dynamics}

Sometimes one is interested in analyzing the behavior of a system composed by a series of microscopic entities (particles assumed with a null extension) dispersed into another fluid (the solvent). The kinematic of such particles depends, of course, on their interactions with the solvent particles. A real molecular dynamics simulation is definitively forbidden (the MD simulation feasibilities nowadays rarely exceeds the number of particles contained within a cube of one micron of side).

One possibility to reduce the size of the discrete models lies in considering only the particles of interest. The other particles (the ones that constitute the solvent) are not considered explicitly and only their averaged effects are retained in the modelling.

Thus, the motion equation of a particle whose position is described by $\mathbf{x}_{i}$, is governed by the Langevin's equation:

$$
m \frac{d^{2} \mathbf{x}_{i}}{d t^{2}}=\xi\left(\frac{d \mathbf{x}_{i}}{d t}-\mathbf{v}_{f}\left(\mathbf{x}_{i}\right)\right)+\mathbf{F}_{i}^{e x t}(t) ; \quad \forall i
$$

where $m$ denotes the particle mass, $\mathbf{x}_{i}$ the position of particle $i, \xi$ the friction coefficient, $\mathbf{v}_{f}\left(\mathbf{x}_{i}\right)$ the fluid velocity at position $\mathbf{x}_{i}$ and $\mathbf{F}_{i}^{e x t}(t)$ all the other forces acting on the particle $i$ (coming from a external potential or from the solvent particles bombardment). We can see that even if this model doesn't incorporate explicitly the solvent particles population, their effects are taken into account from the drift term $\xi\left(\frac{d \mathbf{x}_{i}}{d t}-\mathbf{v}_{f}\left(\mathbf{x}_{i}\right)\right)$ as well as by the impact forces.

In the last expression the drift term is quite standard, however the external forces acting on each particle deserve some additional comment. In what follows and without any detriment of generality we assume that there is no other force than the one coming from the solvent particles bombardment and that the solvent is macroscopically at rest, i.e. $\mathbf{v}_{f}=\mathbf{0}$. The random nature of the interaction force is modelled from a statistical 
distribution function that becomes fully defined as soon as its mean value and its standard deviation are fixed. In respct of the mean value, one expects a null value if the microscopic dynamics is isotropic. Concerning the standard deviation one must proceed within the statistical mechanics framework. In what follows we summarize the main ideas for the derivation of the standard deviation expression.

If we define $B_{\Delta t}$

$$
B_{\Delta t}=\int_{0}^{\Delta t} \frac{F^{e x t}(t)}{m} d t=\sum_{k=1}^{k=p} \frac{F^{e x t}\left(t_{k}\right)}{m} \delta t
$$

where we assume that in $\Delta t$ the particle is subjected to $p$ impacts from the solvent particles, with $p>>1$. These impacts are modelled by a constant force $F^{e x t}\left(t_{k}\right)$ that applies for a time $\delta t(\Delta t=p \delta t)$. By invoking the central limit theorem we conclude that $B_{\Delta t}$ follows a gaussian distribution $\mathcal{N}(0, q \Delta t)$ because the number of impacts scales linearly with $\Delta t$.

Now, to compute $q$, one could integrate the Langevin's equation to obtain the equilibrium velocity distribution $W$ :

$$
W\left(\frac{d x}{d t}, t \rightarrow \infty\right)=\sqrt{\frac{\xi}{m \pi q}} e^{\frac{\xi\left(\frac{d x}{d t}\right)^{2}}{m q}}
$$

that must coincide with the Maxwell-Boltzmann one (canonical ensemble), from which we deduce the expression of $q$ :

$$
q=\frac{2 \xi k_{b} T}{m^{2}}
$$

where $k_{b}$ is the Boltzmann constant and $T$ the absolute temperature.

Thus, the Langevin's equation is fully defined, by writing:

$$
B_{\Delta t}=\int_{0}^{\Delta t} \frac{F^{e x t}(t)}{m} d t=\mathcal{N}\left(0,2 \frac{\xi k_{b} T}{m^{2}} \Delta t\right)
$$

expression that runs also in the presence of other forces like the ones coming from a gradient of a potential or when $\mathbf{v}_{f} \neq \mathbf{0}$.

Thus, one could track the movement of each particle $\mathbf{x}_{i}$ by considering a standard drift term and a random force whose distribution is perfectly known. This stochastic approach has been traditionally used also for solving deterministic models such as the advection-diffusion one, because one could compute some moments of the resulting distribution by tracking a moderate population of particles instead, to discretize the deterministic counterpart of the advection-diffusion model by using one of the standard mesh-based discretization techniques that could involve an excessive number of degrees of freedom in the case of 3D models.

To illustrate this procedure we consider the simplest form of the advection-diffusion equation: 


$$
\frac{\partial C}{\partial t}+\mathbf{v} \cdot \nabla C=D \Delta C
$$

where $C=C(\mathbf{x}, t)$ is the concentration field and $D$ is the so-called diffusion coefficient.

Obviously, this simple parabolic equation could be solved by using any standard technique (finite differences, finite elements, spectral methods, finite volumes, the method of particles, ...), but in what follows we are solving it using a stochastic approach. For this purpose we assume the initial condition represented by $N$-Dirac masses:

$$
C(\mathbf{x}, t=0) \approx C^{0}(\mathbf{x})=\sum_{i=1}^{i=N} c_{i} \delta\left(\mathbf{x}-\mathbf{x}_{i}(t=0)\right)
$$

Here, we are not discussing the choice of the coefficients $c_{i}$ and the locations $\mathbf{x}_{i}$. Several possibilities exist to perform a choice trying to represent, as precisely as possible, the initial concentration distribution. Most of them proceed by regularizing the Dirac distribution and then enforcing the minimization of $\left\|C(\mathbf{x}, t=0)-C^{0}(\mathbf{x})\right\|$. From now on, $c_{i}$ and $\mathbf{x}_{i}(t=0)$ are assumed known. Moreover, as the considered advection-diffusion equation does not contain source terms, the weights $c_{i}$ remain unchanged during the evolution of the pseudo-particles positions $\mathbf{x}_{i}(t)$.

Now, the simplest explicit integration algorithm proceeds by updating the particle position considering both the deterministic and the random contributions:

$$
\mathbf{x}_{i}^{n+1}=\mathbf{x}_{i}^{n}+\mathbf{v}\left(\mathbf{x}_{i}^{n}\right) \Delta t+\mathcal{N}(0,2 D \Delta t) \mathbf{u}, \quad \forall i
$$

where $\mathbf{x}_{i}^{n+1} \equiv \mathbf{x}_{i}(t=(n+1) \times \Delta t)$ and $\mathbf{u}$ is a unit random vector.

Now the distribution moments can be easily computed, and the concentration field could be reconstructed by employing some appropriate smoothing.

It is usual to find this kind of advection-diffusion equation in many branches of science and engineering. In particular they are encountered when one models macromolecular materials within the statistical mechanics framework, as we describe later. Despite its intrinsic simplicity, sometimes they arise defined in highly dimensional spaces including the physical and the conformation coordinates. To avoid the curse of dimensionality drawback characteristic of mesh-based techniques, different authors proposed the use of stochastic techniques exploiting the equivalence between the socalled Fokker-Planck equation and the Ito's stochastic equation [9], similar to the just described equivalence between models (23) and (25). Thus, if one is only interested in computing some moments of the resulting distribution function a moderate population of particles is enough to describe accurately the evolution of such moments. The size of the pseudo-particles population that must be considered for computing accurately the different moments of the solution scales linearly with the dimension of the space, however, if one wants to reconstruct the distribution itself, an impressive number of 
particles is required which do not scale any more linearly with the dimension of the space.

In general the technique just presented, is conceptually very simple and then easy to implement in a computer or in a parallel computing platform. Explicit integration schemes are usually employed, needing a careful choice and control of the time step.

It is nowadays widely recognized that the main drawbacks of stochastic techniques are: (i) the control of the statistical noise that makes the use of the stochastic approach to perform inverse parameter identification or optimization difficult, because the poor accuracy in the sensitivity analysis; (ii) the difficulty in reconstructing the model solution itself even in moderate multidimensional models; and (iii) the necessity of always solving the transient model, even if one is only interested in the steady state.

Moreover, in complex flow simulation using for example a finite element solver for the flow kinematics computation, one must ensure that all the elements contain a number of particles, at least enough to allow computation of the virial stress (the usual micro-macro bridge). Different possibilities exist, but all of them have a non negligible impact on the solution. Thus, if new particles are added (and probably others removed) the size of the model is changing, tracking procedures are time consuming, and the initialization of the just introduced particles induces a noticeable numerical diffusion.

One could think that the aforementioned difficulties could be circumvented by employing a Lagrangian description of the flow combined with a Lagrangian description of the microstructure evolution (stochastic approach), however usual mesh-based strategies fails because of the high distortion of the meshes during the flow. A first tentative attempt at coupling a meshless Lagrangian description of the flow kinematics (using the natural element method widely described in $[10,11]$ ) and a Lagrangian microstructure description have been recently performed in [12]. This technique could be extended for coupling a Lagrangian flow description with a stochastic description of the microstructure evolution. In any case the difficulties just mentioned will persist.

To reduce the computational cost of numerical simulations different model reduction techniques have been proposed recently [13]. However, the coupling of such techniques (based on the proper orthogonal decomposition - also known as KarhunenLoève decomposition) with a Lagrangian description of the microstructure evolution remains nowadays an open problem. However, sometimes the stochastic model can be written in an Eulerian form (Brownian Configuration Fields) and in that case, as illustrated in [14], the model reduction runs are opening some interesting perspectives.

\subsection{Coming back to continuous descriptions: kinetic theory mod- els}

The next level of description concerns the statistical mechanics that we are going to illustrate by considering the dynamics of $N$ electrically charged particles of masse $m$. When $N$ becomes too large, direct molecular dynamics simulations are prohibitive from the computing time viewpoint. Thus, more than describing the system from the 
position and velocities of all the particles, one could introduce the function $f(t, \mathbf{x}, \mathbf{v})$ given the number of particles that at time $t$, are located within an elementary volume $d \mathbf{x}=(d x, d y, d z)^{T}$ placed at position $\mathbf{x}$ and having velocities within the volume defined by $d \mathbf{v}=(d u, d v, d w)^{T}$ around $\mathbf{v}$. Now, the density balance is written:

$$
\frac{\partial f}{\partial t}+\mathbf{v} \cdot \nabla_{x} f+\mathbf{a}(\mathbf{x}, t) \cdot \nabla_{v} f=S(t, \mathbf{x}, \mathbf{v})
$$

where $\nabla_{x}$ and $\nabla_{v}$ represent the gradient operator in the physical and velocity spaces respectively. We assumed that the acceleration $\mathbf{a}=\frac{d \mathbf{v}}{d t}$ does not depend on the velocity (by this reason it is is not affected by the velocity-gradient $\nabla_{v}$ ). The source term $S(t, \mathbf{x}, \mathbf{v})$ represents the so-called collision term and can be derived from an appropriate physical analysis.

We do not need any physics to model the velocity field $\mathbf{v}$ because now the velocity field is a real coordinate, like the spatial ones. On the contrary, we need to define the acceleration field $\mathbf{a}(\mathbf{x}, t)$. For this purpose we consider the Newton's law $\mathbf{a}=\frac{\mathbf{F}}{m}$, and compute the force acting on the particles by taking into account the nature of the system, that in the case addressed here consists of a population of charged particles interacting by means of the Coulomb's potential. The electrostatic potential $U(\mathbf{x}, t)$ can be computed by solving the associated Poisson's problem:

$$
\Delta U(\mathbf{x}, t)=-4 \pi k Q(\mathbf{x}, t)
$$

where $k$ is the Coulomb's law constant and $Q(\mathbf{x}, t)$ is the electrical charge inside the volume $d \mathbf{x}$ around $\mathbf{x}$ that can be computed from:

$$
Q(\mathbf{x}, t)=q \int_{\mathcal{R}^{3}} f(\mathbf{x}, \mathbf{v}, t) d \mathbf{v}
$$

with $q$ the particle charge. The force acting on a particle can be finally computed by using:

$$
\mathbf{F}(\mathbf{x}, t)=-q \nabla_{x} U(\mathbf{x}, t)
$$

\section{Remarks:}

1. When the particles are not charged the steady state solution of Equation (26) leads to the Maxwell-Boltzmann distribution when the appropriate choice of the collision term representing the particles collisions is made.

2. When the interaction potential does not comply with Coulomb's law, model (27)-(29) cannot be employed. In this case we must compute the force by using:

$$
\mathbf{F}(\mathbf{x}, t)=\int_{\mathcal{R}^{3}} \mathbf{F}\left(\mathbf{x}, t ; \mathbf{x}^{\prime}\right) \tilde{f}\left(\mathbf{x}^{\prime}, t\right) d \mathbf{x}^{\prime}
$$


where

$$
\tilde{f}(\mathbf{x}, t)=\int_{\mathcal{R}^{3}} f(\mathbf{x}, \mathbf{v}, t) d \mathbf{v}
$$

and $\mathbf{F}\left(\mathbf{x}, t ; \mathbf{x}^{\prime}\right)$ represents the force at position $\mathbf{x}$ originated by a particle located at position $\mathrm{x}^{\prime}$. In any case this analysis fails when (i) the inter-particle potential leads to a non-definite integral (30); and (ii) in the case of dense systems where the movements of particles is correlated.

3. When the acceleration does not depend on the velocity (as was assumed in Equation (26)) and the collision terms vanish, an equivalence between the conservation equation and the Liouville's theorem in the phase space can be established.

4. In general the establishment of collision terms is quite difficult. To circumvent this difficulty and assuming that the equilibrium distribution is known $f_{e q}(\mathbf{x}, \mathbf{v}, t)$, one could approximate the collision term by

$$
S(t, \mathbf{x}, \mathbf{v})=\frac{f_{e q}(\mathbf{x}, \mathbf{v}, t)-f(\mathbf{x}, \mathbf{v}, t)}{\tau}
$$

where $\tau$ represents a relaxation time. This approximation leads to the so-called BFK models.

5. Equation (26), also known as the Vlasov-Poisson-Boltzmann equation, is widely used to model quantum plasmas. One could imagine the application of this formalism to a variety of physical models: colloids, ferrofluids, coarse grained molecular dynamics, crystallization, etc. Some examples will be presented later and others are in progress. The associated kinetic theory descriptions constitute the Vlasov-Fokker-Planck formalism.

6. The kinetic theory formalism allows for transforming a discrete model into its continuous counterpart. However, in general, the continuous descriptions involve highly multidimensional spaces and their evolutions are governed by hyperbolic non-linear partial differential equations. To solve these kinds of models, appropriate stabilized solvers, able to proceed in highly multidimensional spaces, are needed. We come back to this point later.

In the previous paragraphs we introduced some ideas related to kinetic theory models of systems composed of particles. We are introducing in the next paragraphs some models describing the microscopic modelling of polymeric liquids. For the sake of simplicity we are focusing on polymer solutions (the entangled systems related to the polymer melts can be also described in the kinetic theory framework $[15,16])$. We are addressing the Bead-Spring-Chain (BSC) model of polymer solutions. The BSC chain consists of $S+1$ beads connected by $S$ springs. The bead serves as an interaction point with the solvent and the spring contains the local stiffness information depending on local stretching (see [15] for more details). From now on we are also assuming a fully developed homogeneous flow. Thus, the microstructural state does not depend on the space coordinates. 
The dynamics of the chain are governed by viscous drag, Brownian and connector forces. If we denote by $\dot{\mathbf{r}}_{k}$ the velocity of bead $k$ and by $\dot{\mathbf{q}}_{k}$ the velocity of the spring connector $\mathbf{q}_{k}$, we have

$$
\dot{\mathbf{q}}_{k}=\dot{\mathbf{r}}_{k+1}-\dot{\mathbf{r}}_{k} \quad \forall k=1, \ldots, S
$$

The dynamics of each bead can be written as:

$$
\underbrace{-\zeta\left(\dot{\mathbf{r}}_{k}-\mathbf{v}_{0}-\nabla \mathbf{v} \cdot \mathbf{r}_{k}\right)}_{\text {Viscous drag }}-\underbrace{k_{b} T \frac{\partial \ln (\psi)}{\mathbf{r}_{k}}}_{\text {Brownian effects }}+\underbrace{\mathbf{F}_{k}^{c}-\mathbf{F}_{k-1}^{c}}_{\text {Interaction Forces }}=0,
$$

where $\zeta$ is the drag coefficient, $\mathbf{v}$ is the velocity field, $\mathbf{v}_{0}$ is an average velocity, $k_{b}$ is the Boltzmann constant, $T$ is the absolute temperature and $\psi$ is the distribution function $\psi\left(\mathbf{r}_{1}, \cdots, \mathbf{r}_{S+1}, t\right)$. From equations (33) and (34) we obtain:

$$
\dot{\mathbf{q}}_{k}=\nabla \mathbf{v} \cdot \mathbf{q}_{j}-\frac{1}{\zeta} \sum_{l=1}^{S} \mathbf{A}_{k l} \cdot\left(k_{b} T \frac{\partial \ln (\psi)}{\partial \mathbf{q}_{l}}+\mathbf{F}_{l}^{c}\right),
$$

where $\mathbf{A}_{k l}$ are the components of the Rouse matrix (see [15] for more details).

In the Rouse model the connector force $\mathbf{F}^{c}$ is a linear function of the connector vector, but we could use Finitely Extensible Non Linear springs, with a dimensionless connector force given by:

$$
\mathbf{F}^{c}\left(\mathbf{q}_{k}\right)=\frac{1}{1-\mathbf{q}_{k}^{2} / b} \mathbf{q}_{k}
$$

where $\sqrt{b}$ is the maximum dimensionless length of each spring connector of the chain.

Introducing Equation (35) in the equation governing the evolution of the distribution function, and considering homogeneous flows,

$$
\frac{\partial \psi\left(\mathbf{q}_{1}, \ldots, \mathbf{q}_{S}, t\right)}{\partial t}=-\sum_{k=1}^{S}\left(\frac{\partial}{\partial \mathbf{q}_{k}}\left(\dot{\mathbf{q}}_{k} \psi\left(\mathbf{q}_{1}, \ldots, \mathbf{q}_{S}, t\right)\right)\right)
$$

we obtain

$$
\begin{aligned}
\frac{\partial \psi}{\partial t}=-\sum_{k=1}^{S}( & \left.\frac{\partial}{\partial \mathbf{q}_{k}}\left(\left(\nabla \mathbf{v} \cdot \mathbf{q}_{k}-\frac{1}{\zeta} \sum_{l=1}^{S} \mathbf{A}_{k l} \cdot \mathbf{F}_{l}^{c}\right) \psi\right)\right)+ \\
& +\frac{k_{b} T}{\zeta} \sum_{k=1}^{S} \sum_{l=1}^{S} \mathbf{A}_{k l} \frac{\partial^{2} \psi}{\partial \mathbf{q}_{k} \partial \mathbf{q}_{l}}
\end{aligned}
$$

The micro-macro bridging is performed by computing the virial stress, that within the rheology community is known as the Kramer's rule. The main difficulty in using this description is the highly multidimensional problem defined by Equation (38) that needs for specific advanced solvers such as the ones that which we proposed in some of our former works and we revisit in the next section. 


\section{Advanced solvers for multi-dimensional models}

The solution of models like the one just addressed in Equation (38) needs new strategies, because the standard ones suffer from the curse of dimensionality. Some strategies have been recently proposed for solving models defined in multi-dimensional spaces. The sparse grid techniques are one of the most popular [17], but as we described in the first section, separate representations were also used for solving the models encountered in quantum mechanics (Hartree-Fock and post-Hartree-Fock techniques).

The sparse grids [17] are restricted (as argued in [18]) for treating models involving up to twenty dimensions. On the other hand, separated representations like the one considered in the multi-configuration-self-consistent-fields (MCSCF) [2,3], fix the number of products (anti-symmetrized) and also assume that those products are constructed by combining a certain number of unknown one-dimensional functions.

In the next section we are describing another alternative and efficient technique recently proposed and based on the use of separated representations within a variational framework.

\subsection{A new strategy for circumventing curse of dimensionality by using separated representations}

Recently we proposed a new strategy able to solve highly multi-dimensional models circumventing the curse of dimensionality. This technique was successfully applied for treating some multi-dimensional models encountered in the kinetic theory description of complex fluids $[19,20]$. It allows for defining the optimal number of products containing the optimal one-dimensional functions. In what follows we are summarizing the main ideas that the aforementioned technique involves. For the sake of simplicity, we are illustrating the solution procedure by solving the Poisson problem defined in a space of dimension $D$ :

$$
\triangle T=-f\left(x_{1}, x_{2}, \ldots, x_{D}\right),
$$

where $T$ is a scalar function of $x_{1}, x_{2}, \ldots, x_{D}$. Problem (39) is defined in the domain $\Omega=]-L,+L\left[{ }^{D}\right.$ with vanishing boundary conditions.

The problem solution can be written in the form:

$$
T\left(x_{1}, x_{2}, \ldots, x_{D}\right)=\sum_{j=1}^{\infty} \alpha_{j} \prod_{k=1}^{D} F_{k j}\left(x_{k}\right)
$$

where $F_{k j}$ is the $j^{t h}$ approximation function, with unit norm and that depends only on the $k^{\text {th }}$ coordinate. 
It is well known that the solution of numerous problems can be accurately approximated using a finite (sometimes very reduced) number of approximation functions, i.e.:

$$
T\left(x_{1}, x_{2}, \ldots, x_{D}\right) \approx \sum_{j=1}^{Q} \alpha_{j} \prod_{k=1}^{D} F_{k j}\left(x_{k}\right)
$$

The previous expression implies the same number of approximation functions in each dimension, but each one of these functions could be expressed in a discrete form using a different number of parameters (nodes of the one-dimensional grids).

Now, an appropriate numerical procedure is needed for computing the coefficients $\alpha_{j}$ as well as the $Q \times D$ one-dimensional approximation functions. The proposed numerical scheme consists of an iteration procedure that solves at each iteration $n$ the following three steps:

Step 1: Projection of the solution in a discrete basis

If we assume the functions $F_{k j}, \forall j \in[1, \ldots, n] ; \forall k \in[1, \ldots, D]$ known (verifying the boundary conditions), the coefficients $\alpha_{j}$ can be computed by introducing the approximation of $T$ into the Galerkin variational formulation associated with Equation (39):

$$
\int_{\Omega} \nabla T^{*} \cdot \nabla T d \Omega=\int_{\Omega} T^{*} f d \Omega
$$

Introducing the approximation of $T$ and $T^{*}$ :

$$
\begin{aligned}
T\left(x_{1}, x_{2}, \ldots, x_{D}\right) & =\sum_{j=1}^{n} \alpha_{j} \prod_{k=1}^{D} F_{k j}\left(x_{k}\right), \\
T^{*}\left(x_{1}, x_{2}, \ldots, x_{D}\right) & =\sum_{j=1}^{n} \alpha_{j}^{*} \prod_{k=1}^{D} F_{k j}\left(x_{k}\right),
\end{aligned}
$$

into Equation (42), we have

$$
\begin{gathered}
\int_{\Omega} \nabla\left(\sum_{j=1}^{n} \alpha_{j}^{*} \prod_{k=1}^{D} F_{k j}\left(x_{k}\right)\right) \cdot \nabla\left(\sum_{j=1}^{n} \alpha_{j} \prod_{k=1}^{D} F_{k j}\left(x_{k}\right)\right) d \Omega= \\
=\int_{\Omega}\left(\sum_{j=1}^{n} \alpha_{j}^{*} \prod_{k=1}^{D} F_{k j}\left(x_{k}\right)\right) f d \Omega
\end{gathered}
$$

Now, we assume that $f\left(x_{1}, \cdots, x_{D}\right)$ can be written in the form 


$$
f\left(x_{1}, \cdots, x_{D}\right)=\sum_{h=1}^{m} \prod_{k=1}^{D} f_{k h}\left(x_{k}\right) .
$$

Equation (45) involves integrals of products of $D$ functions each one defined in a different dimension. Let $\prod_{k=1}^{D} g_{k}\left(x_{k}\right)$ be one of these functions to be integrated. The integral over $\Omega$ can be performed by integrating each function in its definition interval and then multiplying the $D$ computed integrals according to:

$$
\int_{\Omega} \prod_{k=1}^{D} g_{k}\left(x_{k}\right) d \Omega=\prod_{k=1}^{D} \int_{-L}^{L} g_{k}\left(x_{k}\right) d x_{k}
$$

which makes possible the numerical integration in highly dimensional spaces.

Now, due to the arbitrariness of the coefficients $\alpha_{j}^{*}$, Equation (45) allows computation of the $n$-approximation coefficients $\alpha_{j}$, solving the resulting linear system of size $n \times n$. This problem is linear and moreover rarely exceeds the order of tens of degrees of freedom in standard elliptic models. Thus, even if the resulting coefficient matrix is densely populated, the time required for its solution is negligible with respect to the one required for performing the approximation basis enrichment (step 3).

\section{Step 2: Checking convergence}

From the solution of $T$ at iteration $n$ given by Equation (43) we compute the residual $R e$ related to Equation (39):

$$
R e=\frac{\sqrt{\int_{\Omega}\left(\triangle T+f\left(x_{1}, \cdots, x_{D}\right)\right)^{2}}}{\|T\|} .
$$

The integral in Equation (48) can be written as the product of one-dimensional integrals by performing a separated representation of the square of the residual.

If $R e<\epsilon$ (epsilon is a small enough parameter) the iteration process stops, yielding the solution $T\left(x_{1}, \cdots, x_{D}\right)$ given by Equation (43). Otherwise, the iteration procedure continues.

\section{Step 3: Enrichment of the approximation basis}

From the coefficients $\alpha_{j}$ just computed the approximation basis can be enriched by adding the new function $\prod_{k=1}^{D} F_{k(n+1)}\left(x_{k}\right)$. For this purpose we solve the non-linear Galerkin variational formulation related to Equation (39):

$$
\int_{\Omega} \nabla T^{*} \cdot \nabla T d \Omega=\int_{\Omega} T^{*} f d \Omega
$$


using the approximation of $T$ given by:

$$
T\left(x_{1}, x_{2}, \ldots, x_{D}\right)=\sum_{j=1}^{n} \alpha_{j} \prod_{k=1}^{D} F_{k j}\left(x_{k}\right)+\prod_{k=1}^{D} R_{k}\left(x_{k}\right) .
$$

The solution of Equation (49) can be expressed from the stationarity of functional $J(T)$ :

$$
J(T)=\int_{\Omega} \frac{1}{2}(\nabla T)^{2} d \Omega-\int_{\Omega} T f d \Omega
$$

which results in:

$$
\delta J=\int_{\Omega} \nabla \delta T \cdot \nabla T d \Omega-\int_{\Omega} \delta T f d \Omega=0,
$$

that corresponds to Equation (49) by putting $\delta T=T^{*}$.

Now, taking the variation of $T$ according to its expression given by Equation (50), where the variation of the known functions $F_{k j}$ vanishes, we have:

$$
\begin{gathered}
\delta T=\delta\left(R_{1}\left(x_{1}\right) \cdots R_{D}\left(x_{D}\right)\right)= \\
=\delta R_{1}\left(x_{1}\right) R_{2}\left(x_{2}\right) \cdots R_{D}\left(x_{D}\right)+\cdots+R_{1}\left(x_{1}\right) R_{2}\left(x_{2}\right) \cdots \delta R_{D}\left(x_{D}\right),
\end{gathered}
$$

that can be written as

$$
\begin{gathered}
T^{*}\left(x_{1}, x_{2}, \ldots, x_{D}\right)= \\
=R_{1}^{*}\left(x_{1}\right) R_{2}\left(x_{2}\right) \cdots R_{D}\left(x_{D}\right)+\cdots+R_{1}\left(x_{1}\right) R_{2}\left(x_{2}\right) \cdots R_{D}^{*}\left(x_{D}\right) .
\end{gathered}
$$

leading to a non-linear variational problem, whose solution is the $D$ functions $R_{k}\left(x_{k}\right)$, $k=1, \cdots, D$. After convergence of the non-linear solver, functions $F_{k(n+1)}\left(x_{k}\right)$ are finally obtained by normalizing the functions $R_{1}, R_{2}, \ldots, R_{D}$.

The discretization needs an appropriate discrete approximation of functions $R_{k}\left(x_{k}\right)$ and $F_{k j}\left(x_{k}\right)$. Each one of these functions is approximated using a 1D finite element description. If we assume than $p_{k}$ nodes are used to construct the interpolation of functions $R_{k}\left(x_{k}\right)$ and $F_{k j}\left(x_{k}\right)$ in the interval $[-L, L]$, then the size of the resulting discrete non-linear problem is $\sum_{k=1}^{k=D} p_{k}$. The price to pay for avoiding a whole mesh in the multidimensional domain is the solution of a non-linear problem whose size scales linearly with the dimension of the space. However, even in high dimensions the size of the non-linear problems remain moderate and no particular difficulties have been found in its solution.

Concerning the computation time, even when the non-linear solver converges quickly, this step consumes the main part of the global computing time. Different non-linear solvers have been analyzed: fixed-point, Newton or one based on an alternating directions scheme [19-21].

Preliminary convergence analysis reveals that even when one uses piecewise linear one-dimensional finite elements to approximate all the one-dimensional functions involved by the separated representation, the convergence rate (in the $\mathcal{L}^{2}$-norm) seems 
to be fourth order. This result is a little bit strange because we expected the usual second order convergence. We are performing further analysis to check if there is some super-convergence phenomena. At the same time one could modify the onedimensional approximations to enhance the approximation consistency and then the convergence rates by using spectral approximations or use wavelet approximation basis to take advantage of its multi-resolution properties, specially useful to define adaptive multi-scale strategies. Another possibility to enhance the convergence rate could be the use of moving least squares -MLS- based approximations (widely used in the context of meshless methods [22,23]).

\section{Conclusions}

In this paper we presented different physical descriptions at different scales involved in material science. At the lowest scale the quantum mechanics involves high-dimensional models whose solution, when fermions are considered, must be anti-symmetric. We have pointed out that in fact more than the multidimensional character of quantum mechanics models, the real challenge is the anti-symmetry constraint. All the practical details concerning the discretization of the Schrödinger equation by using a wavefunction separated representation can be found in [24]. However, when the solution of the Schrödinger equation is addressed a new difficulty appears, the one related to the Pauli's principle that requires the anti-symmetrization of each one of the functional products involved by the separated representation. This anti-symmetrization is performed by using the Slater's determinants, but we must recall that the complexity in the use of such determinants scales in the order of $N$ !, being $N$ the number of electrons involved in the quantum system. In this way, we can affirm that the main difficulty in the solution of the Schrödinger equation is not its multi-dimensional character, but the necessity of enforcing the solution anti-symmetry when fermions are involved.

Thus, at present, the procedure just employed must be viewed as an enhanced variant of the MCSCF (multi-configuration self consistent field) technique that considers an optimal number of determinants and the optimal functions (in general all the functions involved by the different anti-symmetrized products -determinants- are different).

Coarser modelling involves molecular dynamics or Brownian dynamics but despite its conceptual simplicity its use is not exempted from computational and conceptual difficulties. The next level is the one that corresponds to statistical mechanics descriptions leading to continuous models described by non-linear and coupled multidimensional partial differential equations. Some of these models have been successfully solved in some of our former works $[19,20]$ by using the adaptive separated representation revisited in the present work. This technique seems a promising alternative for addressing highly-multidimensional models.

The main perspectives of this work are: (i) the possibility of performing a kinetic theory description of different models until now described in the context of molec- 
ular dynamics; (ii) the improving of separated representation by enhancing the convergence rate, by enriching the approximation basis and by stabilizing the advection terms that those models usually involve; and (iii) the exploration of new quantum mechanics descriptions as the ones derived from the Dirac formalism or by applying the Schrödinger one to systems composed of bosons where the Pauli's principle do not apply.

\section{References}

[1] R.B. Laughlin, "The theory of everything”, Proceeding of the U.S.A. National Academy of Sciences, 2000.

[2] E. Cancès, M. Defranceschi, W. Kutzelnigg, C. Le Bris, Y. Maday, "Computational Quantum Chemistry: a primer”, Handbook of Numerical Analysis, Vol. X, Elsevier, 3-270, 2003.

[3] "Handbook of numerical analysis, Vol. X: Computational chemistry", C. Le Bris editor, Elsevier, 2003.

[4] V. Gavini, K. Bhattacharya, M. Ortiz, "Quasi-continuum orbital-free densityfunctional theory: A route to multi-million atom non-periodic DFT calculation”, Journal of the Mechanics and Physics of Solids, 55/4, 697-718, 2007.

[5] V.B. Shenoy, R. Millera, E.B. Tadmor, D. Rodney, R. Phillips, M. Ortiz, "An adaptive fnite element approach to atomic-scale mechanics - the quasicontinuum method", Journal of the Mechanics and Physics of Solids, 36, 500-531, 1999.

[6] G.J. Wagner, W.K. Liu, "Coupling of atomistic and continuum simulations using a bridging scale decomposition”, Journal of Computational Physics, 190, 249274, 2003.

[7] H. Ben Dhia, "Multiscale mechanical problems: the Arlequin method", C. R. Acad. Sci., Paris 326, Ser-II b, 899-904, 1998.

[8] S.P. Xiao, T. Belytschko, "A bridging domain method for coupling continua with molecular dynamics”, Comput. Methods Appl. Mech. Engrg., 193, 1645-1669, 2004.

[9] H.C. Öttinger, M. Laso, "Smart polymers in finite element calculation”, In P. Moldanaers and R. Keunings (Editors) Theoretical and Applied Rheology, Vol. 1, Proceedings of the XIth International Congress on Rheology (Elsevier), 286288, 1992.

[10] M.A. Martinez, E. Cueto, I. Alfaro, M. Doblare, F. Chinesta, "Updated Lagrangian free surface flow simulations with the natural neighbour Galerkin methods", International Journal for Numerical Methods in Engineering, 60/13, 2105-2129, 2004.

[11] D. Gonzalez, E. Cueto, F. Chinesta, M. Doblare, “An updated Lagrangian strategy for free-surface fluid dynamics”, Journal of Computational Physics, 223, 127-150, 2007.

[12] M.A. Martinez, E. Cueto, M. Doblare, F. Chinesta, "Natural element meshless 
simulation of injection processes involving short fiber suspensions", Journal of Non-Newtonian Fluid Mechanics, 115, 51-78, 2003.

[13] A. Ammar, D. Ryckelynck, F. Chinesta, R. Keunings, "On the reduction of kinetic theory models related to finitely extensible dumbbells", 134, 136-147, 2006.

[14] F. Chinesta, A. Ammar, A. Falco, M. Laso, "On the reduction of stochastic kinetic theory models of complex fluids", Modelling and Simulation in Material Science and Engineering, 15, 639-652, 2007.

[15] B.B. Bird, C.F. Curtiss, R.C. Armstrong, O. Hassager, "Dynamics of polymeric liquids", vol. 2, John Wiley \& Sons, 1987.

[16] R.G. Larson, "The structure and rheology of complex fluids”, Oxford University Press, New York, 1999.

[17] H.J. Bungartz, M. Griebel, "Sparse grids”, Acta Numer., 13, 1-123, 2004.

[18] Y. Achdou, O. Pironneau, “Computational methods for option pricing”, SIAM Front. Appl. Math., 2005.

[19] A. Ammar, B. Mokdad, F. Chinesta, R. Keunings, "A new family of solvers for some classes of multidimensional partial differential equations encountered in kinetic theory modeling of complex fluids", J. Non-Newtonian Fluid Mech., 139, 153-176, 2006.

[20] A. Ammar, B. Mokdad, F. Chinesta et R. Keunings, "A new family of solvers for some classes of multidimensional partial differential equations encountered in kinetic theory modeling of complex fluids. Part II: transient simulation using space-time separated representations”, J. Non-Newtonian Fluid Mech., 144, 98121, 2007.

[21] G. Beylkin, M. Mohlenkamp, “Algorithms for numerical analysis in high dimensions”, SIAM J. Sci. Commun., 26/6, 2133-2159, 2005.

[22] B. Nayroles, G. Touzot, P. Villon, "Generalizing the finite element method: diffuse approximation and diffuse elements", Computational Mechanics, 10, 307318, 1992.

[23] T. Belytschko, Y.Y. Lu, L. Gu, “Element-free Galerkin methods”, International Journal for Numerical Methods in Engineering, 37, 229-256, 1994.

[24] A. Ammar, F. Chinesta, "Circumventing curse of dimensionality in the solution of highly multidimensional models encountered in quantum mechanics using meshfree separated representations", Lecture Notes on Computational Science and Engineering, Springer, In press. 